\title{
Controlling Polymer Morphologies by Intramolecular and Intermolecular Dynamic Covalent Iron(III)/Catechol Complexation - From Polypeptide Single Chain Nanoparticles to Hydrogels
}

Marco Hebel ${ }^{1 f}$, Jasmina Gačanin ${ }^{1,2} f$, Thorsten Lückerath ${ }^{1,2}$, David $Y$. W. $\mathrm{Ng}^{2 *}$, Tanja Weil ${ }^{1,2 *}$

${ }^{1}$ Marco Hebel, Jasmina Gačanin, Dr. Thorsten Lückerath, Prof. Dr. Tanja Weil

Institute of Inorganic Chemistry I, Ulm University, Albert-Einstein-Allee 11, 89081 Ulm, Germany

E-mail: Tanja.Weil@uni-ulm.de

${ }^{2}$ Jasmina Gačanin, Dr. Thorsten Lückerath, Dr. David Y. W. Ng, Prof. Dr. Tanja Weil

Max Planck Institute for Polymer Research, Ackermannweg 10, 55128 Mainz, Germany

E-mail: david.ng@mpip-mainz.mpg.de,weil@mpip-mainz.mpg.de

${ }^{f}$ Both authors contributed equally

Keywords: Dynamic covalent materials, single chain folding, albumin nanoparticle, albumin hydrogel, stimulus-responsiveness, iron complex.

Abstract: Responsive biomaterials, tunable from the molecular to the macroscopic scale, are attractive for various applications in nanotechnology. Herein, a long polypeptide chain derived from the abundant serum protein human serum albumin was cross-linked by dynamiccoordinative iron(III)/catechol bonds. By tuning the binding stoichiometry and the $\mathrm{pH}$, reversible intramolecular folding into polypeptide nanoparticles with controllable sizes was achieved. Moreover, upon varying the stoichiometry, intermolecular cross-links became predominant yielding smart and tunable macroscopic protein hydrogels. By adjusting the intraand intermolecular interactions, biocompatible and biodegradable materials were formed with varying morphologies and dimensions covering several lengths scales featuring rapid gelation without toxic reagents, fast and autonomous self-healing, tunable mechanical properties and high adaptability to local environmental conditions. Such material characteristics could be particularly attractive for tissue engineering approaches to recreate soft tissues matrices with highly customizable features in a fast and simple fashion. 


\section{Introduction}

Nature's control of supramolecular systems across structural hierarchies from the molecular to the macroscopic range ubiquitously drives cellular processes and structure formation. Controlled supramolecular interactions form the basis for biorecognition as they allow dynamic binding and dissociation based on concentration gradients or environmental stimuli. In various aspects, polymer chemists have taken a great deal of inspiration from Nature, i.e. in creating supramolecular nanostructures of distinct shapes by single chain folding, which mimicks folding processes of proteins or their assembly into larger superstructures. ${ }^{[1-3]}$ There is still great interest to expand the available chemical tools to control folding of a single polymer chain into nano-objects of distinct shapes and to lock these shapes by covalent cross-linking via condensation reactions, cycloaddition reactions (click chemistries), or dynamic covalent bonds such as hydrazones. ${ }^{[1,4,5]}$. In fact, dynamic covalent (DC) bonds play a crucial role in cellular life as they provide many attractive features such as controlled structure formation across hierarchies, stability in aqueous media and reversibility in response to external stimuli such as $\mathrm{pH}$ or redox environments. ${ }^{[6]}$ For instance, disulfides stabilize proteins in their bioactive three dimensional shapes and reversible unfolding i.e. denaturation facilitates digestion and cellular recycling. ${ }^{[7]}$ Apart from stabilization of the folded polypeptide chain at specific positions in the proteins structure, proteins could also be cross-linked by disulfides into the macroscopic fibrillar structures such as in keratin, which provides mechanic stability. ${ }^{[8-10]}$ Dynamic exchange of disulfide bonds proceeds either through enzymes like the protein disulfide isomerases and thioredoxins or in redox environments, allowing Nature to manipulate bioarchitectures on demand. The ingenuity of controlling such a large repertoire of protein structures and assemblies by a single type of DC bond formation indicates that there is still great potential to apply such methodes also for structure formation of polymeric materials in the synthetic world. ${ }^{[11,12]}$ 
In this respect, DC reactions based on metal coordination chemistry offer many exciting features such as stable complex formation in physiological aqueuous media, reversibility as well as the opportunity to record changes in the coordination sphere by optical readout. Due to the presence of a large coordination sphere and the variable oxidation states, metals such as iron(II)/iron(III) can often offer a higher level of design complexity. Catechols, for example, bind to iron(III) in a bidentate format in an octahedral geometry where mono-, bis- and triscatechol coordination is determined simply by $\mathrm{pH}$. Compared to many other reversible motifs such as synthetic hydrogen bonding units, e.g. boronic acid/diol complexation, the association constants of iron(III)/catechol complexes in buffer are several orders of magnitude higher in the range of $\log K_{a} \sim 50 .{ }^{[13-16]}$ As such, the iron(III)/catechol interaction offers distinct advantages for the formation of traceable and reversible DC complexes that remain stable even under physiological conditions.

Inspired by the versatility of DC bonds in Nature, we report the formation of polypeptide nanoparticles that are converted into hydrogels through dynamic covalent complex formation. The polypeptide sequence of the abundant blood plasma protein human serum albumin (HSA) was modified by introducing catechol groups as new dynamic interaction points so that the polypeptide backbone folds intramolecularly into polypeptide nanoparticles (Figure 1). By varying the iron(III)/catechol stoichiometry and/or $\mathrm{pH}$, or concentration, a hydrogel was formed. In this way, we show that the integration of coordination chemistry into macromolecular architectures provides a versatile toolbox that allows facile access to tuneable biomaterials or nanostructures, even under physiological conditions. These biocompatible and biodegradable materials could be of interest for various applications in biological systems due to their rapid gelation without toxic reagents, fast and autonomous self-healing, tunable mechanical properties and high adaptability to local environmental conditions. Such characteristics could be particularly attractive for tissue engineering approaches to recreate different soft matrices in a fast and simple fashion without the necessity to modify the system. 


\section{Results and Discussion}

The HSA protein provides a long polypeptide sequence with high numbers of reactive lysine, aspartic and glutaminc acid groups as well as 35 cysteines that could be easily modified. Moreover, globular HSA could be denatured easily in the presence of chaotropic agents and under reductive conditions. ${ }^{[17,18]}$ HSA was first cationized with ethylenediamine and PEGylated with $\mathrm{PEG}_{2000}-\mathrm{NHS}$ ester $(\sim 2000 \mathrm{~g} / \mathrm{mol})$ in order to stabilize the protein backbone and to prevent aggregate formation and precipitation during disulfide reduction as reported previously. ${ }^{[17,18]}$ Characterized by MALDI-TOF MS, an average of $22 \mathrm{PEG}_{2000}-\mathrm{NHS}$ ester were attached to HSA (Supporting Information).

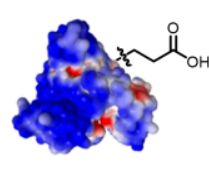

HSA
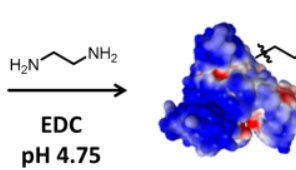

cHSA

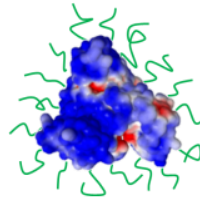

CHSA-PEG

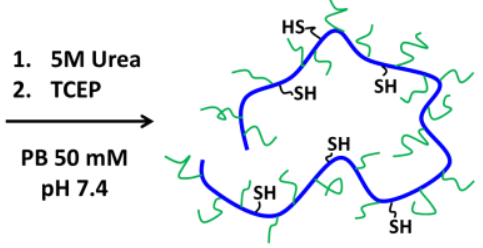

dcHSA-PEG
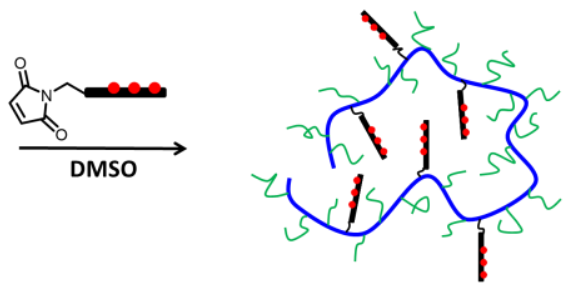

dcHSA-PEG-C3
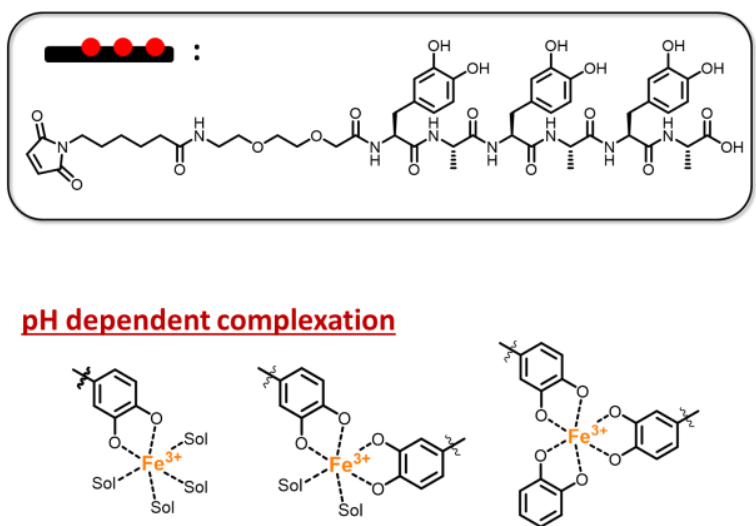

$\mathrm{pH} 4$

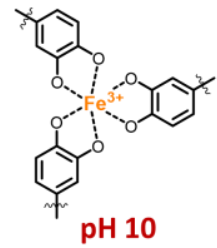

$\mathrm{pH} 7.4$

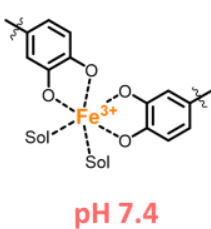

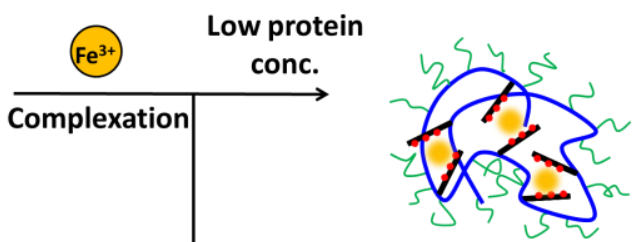
$\underbrace{\mathrm{nm}} \begin{gathered}\text { Protein-folded } \\ \text { nanoparticle }\end{gathered}$
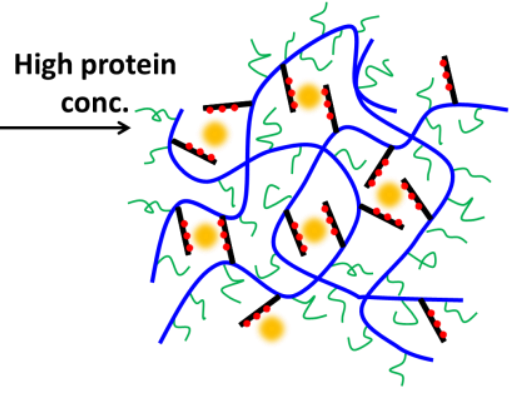

Cross-linked hydrogel

Figure 1. Synthesis route to the biopolymer dcHSA-PEG-C3 and the resulting formation of protein-folded nanoparticles or cross-linked protein hydrogels, $\mathrm{pH}$ dependent coordination of iron(III) to the of catechol groups in aqueous solution. 
Denaturation of the protein backbone by $5 \mathrm{M}$ urea and tris(2-carboxy-ethyl)phosphine (TCEP) ${ }^{[18]}$ exposes the reduced sulfhydryl groups of the cysteine residues for subsequent thiolMichael addition with $\mathbf{C 3}$, a maleimide-terminated oligopeptide with three catechol repeats that serves as anchor groups for the formation of DC metal complexes. The new catechol units are well-positioned at the locations of the 35 cysteine groups due to the chemoselectivity of the maleimide-thiol reaction in polar organic solvents. ${ }^{[19,20]}$ After purification by size exclusion chromatography, dcHSA-PEG-C3 was isolated and functionalization was quantified by applying the fluorescence chemosensor, 2-anthraceneboronic acid, whose interaction with catechols results in quenching of its fluorescence, which was used to determine the number of C3 per dcHSA-PEG (Supporting Information). A maximum of 18 sulfhydryl groups were functionalized by applying $\mathbf{C 3}$ in excess, corresponding to approximately 54 catechol groups attached to the HSA amino acid sequence (Figure $2 \mathbf{b}$ ).

Coordination of iron(III) to the catechol functionalized biopolymer dcHSA-PEG-C3 was assessed by UV-Vis absorption spectroscopy for varying complex stoichiometries and $\mathrm{pH}$ values (Figure 2d). Different amounts of iron(III) (conc. $3.1 \mathrm{mM}$ ) were titrated to dcHSAPEG-C3 at pH 4, 7.4, and 10 (Figure S1, S2, S3). At pH 10, a characteristic absorbance of the tris-catechol iron(III) complex $\left[\mathrm{Fe}(\mathrm{cat})_{3}\right]^{3-}$ was detected at $\sim 475 \mathrm{~nm}^{[21]}$ At $\mathrm{pH} 7.4$, the predominant catechol-iron(III) species $\left[\mathrm{Fe}(\mathrm{cat})_{2}\right]^{-}$appeared with an absorption at $540 \mathrm{~nm}$, along with a minor presence of $\left[\mathrm{Fe}(\mathrm{cat})_{3}\right]^{3-}$. The small shoulder at $710 \mathrm{~nm}$ indicated the presence of a monovalent complex $[\mathrm{Fe}(\mathrm{cat})]^{+} .{ }^{[21,22]}$ At $\mathrm{pH} 4$, no prominent peaks were observed in the visible range. These observations are consistent with experiments on different catechol-iron species in the literature, whose proportions change dynamically with $\mathrm{pH} .{ }^{[21]}$ In order to control complex formation both intra- and intermolecularly, the interaction and coordination extent between iron(III) and catechols on the protein was optimized by UV-vis spectroscopy. By varying the amount of iron(III) from $0-360$ mol equiv., the highest absorbance at $540 \mathrm{~nm}$ was recorded at 180 equiv. indicating the majority of iron(III) existing as bis-catechol iron(III) 
complexes (Figure S3). At 360 mol equiv. of iron(III), the equilibrium of the iron-catechol complexes shifts slightly towards the monovalent complex as excess iron(III) competes for coordination thereby reducing the absorbance of the bis-complex (Figure S3).

a)

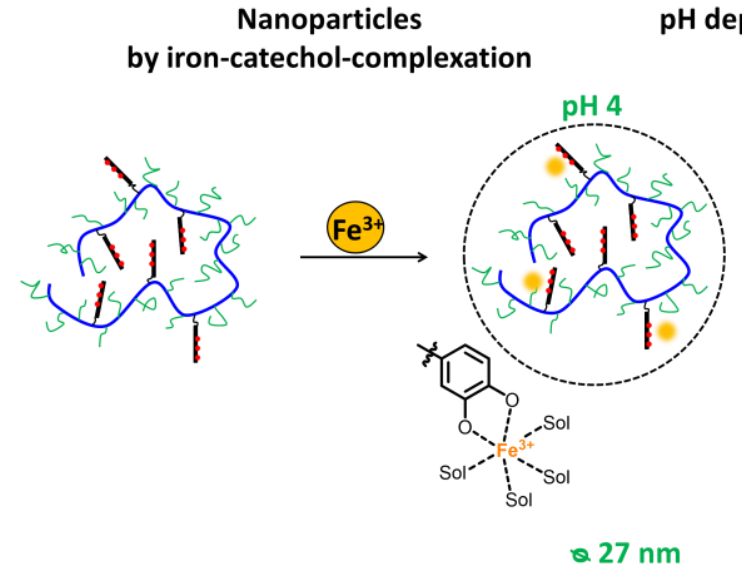

$\mathrm{pH}$ dependent single polymer folding ol-complexation

b)

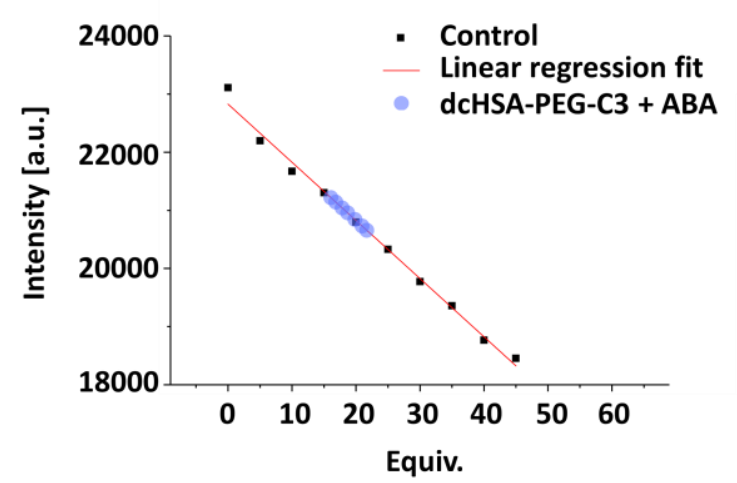

d)

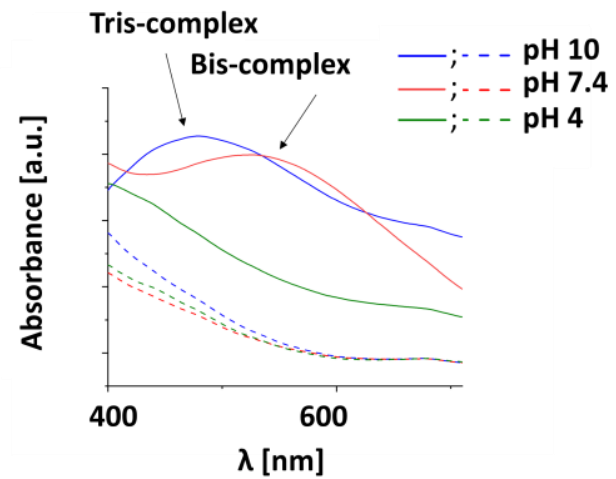

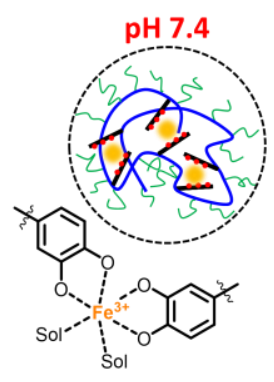

Q. $25 \mathrm{~nm}-19 \mathrm{~nm}$

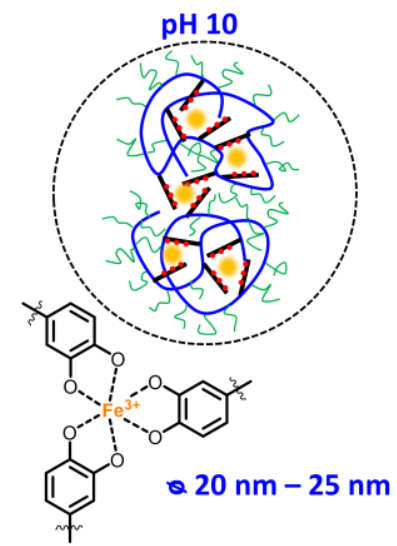

c)

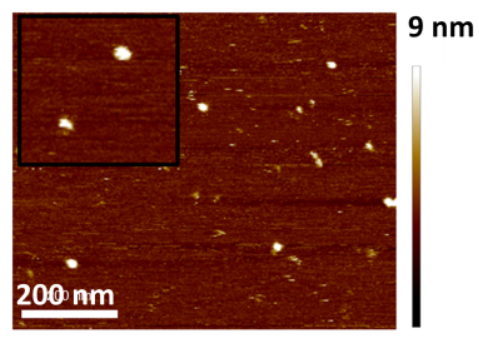

e)

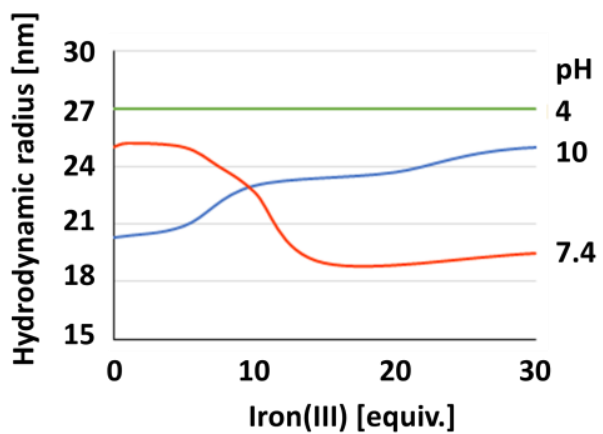

Figure 2. a) Visualisation of dcHSA-PEG-C3 of the single polymer folding process with $\mathrm{pH}$ dependent folding of nanoparticles by iron-catechol-complexation. Under non-complexation conditions at $\mathrm{pH} 4$, no change in the hydrodynamic diameter occurrs regardless of the quantity of the iron(III). However, at $\mathrm{pH} 7.4$ the quantity of added iron(III) determines the nanoparticle size, whereas at $\mathrm{pH} 10$ inter-particle interactions are promoted. b) Measurement of the amount of catechol functionalities on dcHSA-PEG-C3 with an 2-anthracene boronic acid chemosensor assay. The $\mathrm{x}$-axis shows the equivalents of the catechol groups per molecule dcHSA-PEG-C3. The experiment was repeated several times resulting in an average of 18 catechol peptide 
molecules (54 catechol groups) per molecule dcHSA-PEG-C3. c) AFM image of dcHSAPEG-C3 nanoparticles. d) UV-Vis absorbance spectra of dcHSA-PEG-C3 mixed with iron(III) at different $\mathrm{pH}$ values (blue: $\mathrm{pH} 10$, red: $\mathrm{pH} 7.4$, green: $\mathrm{pH} 4$, dashed lines: 0 equiv. iron(III), solid lines: 30 equiv. iron(III)). e) Correlation of the hydrodynamic radius of dcHSA-PEG-C3 with the amount of iron(III) at $\mathrm{pH} 4,7.4$ and 10, measured by DLS.

Under very dilute conditions $(0.7 \mathrm{nM})$, we expect the system to favor intramolecular complexation to afford single chain nanoparticles (Figure 2). In order to reduce secondary interactions of the polypeptide backbone, complexation of iron(III) was accomplished in $8 \mathrm{M}$ urea. To investigate the folding process, different amounts of iron(III) were added into a solution of dcHSA-PEG-C3 $(0.7 \mathrm{nM})$ at $\mathrm{pH} 4,7.4$ and 10 . The formation of single chain polypeptide nanoparticles was monitored by dynamic light scattering (DLS) and size-exclusion chromatography (SEC) (Figure 3). Under non-complexation conditions at $\mathrm{pH} 4$, there was no change in the hydrodynamic diameter of $27 \pm 0.1 \mathrm{~nm}$ regardless of the quantity of the iron(III) added. At pH 7.4, the hydrodynamic diameter of dcHSA-PEG-C3 revealed a gradual reduction from $25 \pm 0.7 \mathrm{~nm}$ (for iron(III) $>7.5 \mathrm{~mol}$ equiv.) to $19 \pm 0.5 \mathrm{~nm}$ (for $30 \mathrm{~mol}$ equiv., Figure 3, S4). This suggests that at least $7.5 \mathrm{~mol}$ equiv. of iron(III) were required to produce a detectable change in the hydrodynamic size of the polypeptide. At 30 mol equiv. of iron(III), full coordination to the catechol groups was achieved. Comparatively, at $\mathrm{pH} 10$, the size distribution increased by $5 \pm 0.5 \mathrm{~nm}$ with increasing amounts of iron(III), promoting inter-particle interactions due to the increase in coordination number.

These size differences observed in DLS were subsequently verified independently using sizeexclusion chromatography (Figure 3d, 3e). At pH 7.4, the retention time of dcHSA-PEG-C3 was successively increased from $28 \mathrm{~min}$ to $29 \mathrm{~min}$ upon the addition of 0 equiv. to 30 equiv. of iron(III). This correlation suggests that the addition of higher amounts of iron(III) reduces the hydrodynamic volume of the nanoparticles. At $\mathrm{pH}$ 10, the nanoparticles showed a larger size and hence a reduction in the retention time from $30 \mathrm{~min}$ to $28.4 \mathrm{~min}$ with increasing amounts of iron(III), complementing the DLS measurements. However, at $\mathrm{pH} 4$, the conjugate was 
retained on the column and could not be eluted most likely due to the interaction between the mono-catechol-iron(III) complexes and the dextran material of the column.

a)

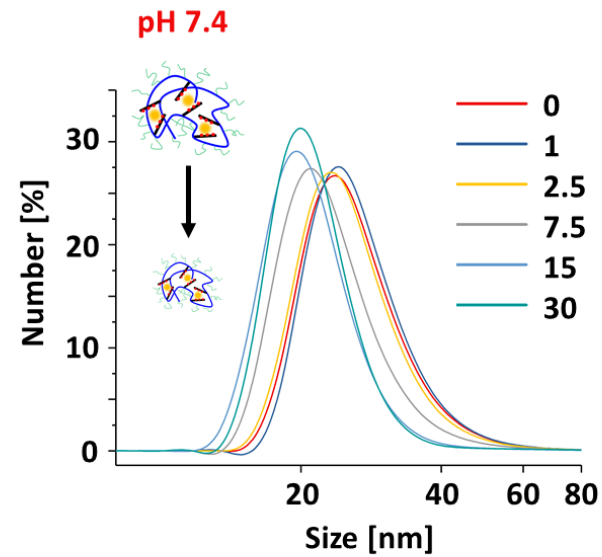

c)

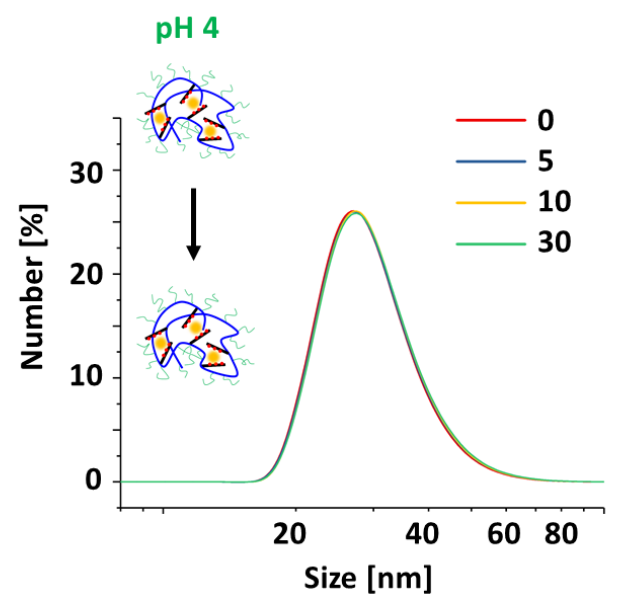

b)

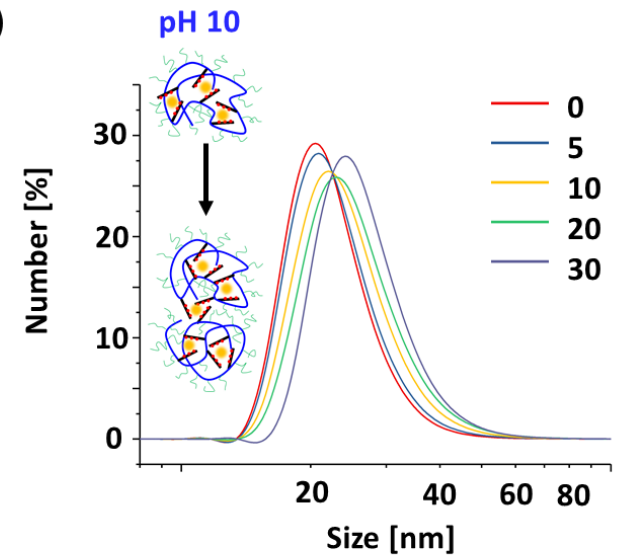

d)

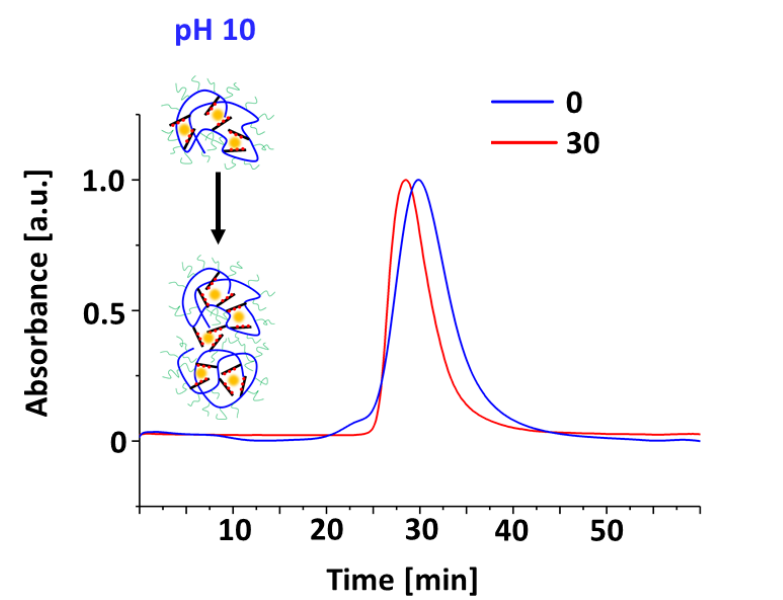

e)

pH 7.4
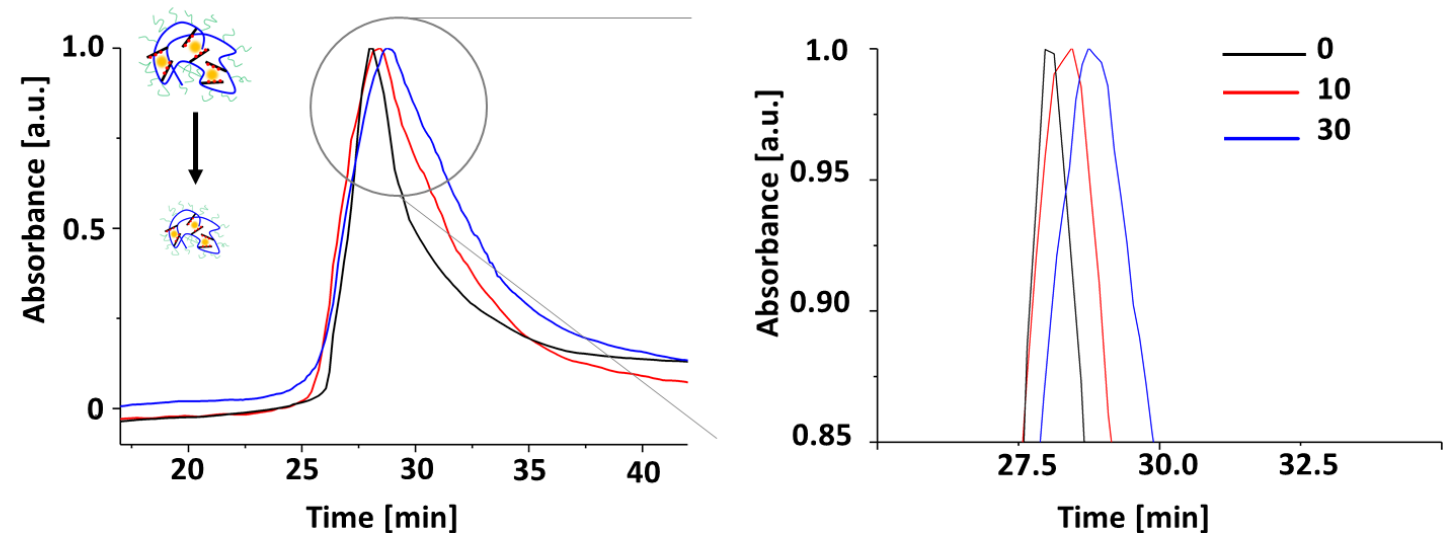

f)

\begin{tabular}{|c|ccc|ccc|}
\hline & \multicolumn{3}{|c|}{ Size distribution [nm] by DLS } & \multicolumn{3}{c|}{ Retention time [min] by SEC } \\
\cline { 2 - 7 } Equiv. iron(III) & 0 & 10 & 30 & 0 & 10 & 30 \\
pH 4 & $27 \pm 0$ & $27 \pm 0.1$ & $27 \pm 0.1$ & - & - & - \\
pH 7.4 & $25 \pm 0.7$ & $22 \pm 0.7$ & $19 \pm 0.5$ & 27.95 & 28.35 & 28.97 \\
pH 10 & $20 \pm 0.1$ & $23 \pm 0.5$ & $25 \pm 0.4$ & 28.42 & - & 29.92 \\
\hline
\end{tabular}

Figure 3. Hydrodynamic size distributions obtained by DLS for dcHSA-PEG-C3 with different amounts of iron(III) (equiv.) at a) $\mathrm{pH} 7.4$, b) $\mathrm{pH} 10$ and c) $\mathrm{pH}$ 4. SEC chromatograms 
for dcHSA-PEG-C3 with different amounts of iron(III) (equiv.) at d) pH 10 and e) pH 7.4, f) table of nanoparticle size distributions and retention times, measured by DLS and SEC.

Taken together, the results of the DLS and SEC indicate that the observed reduction in the hydrodynamic volume of dcHSA-PEG-C3 by iron(III)/catechol complexation originates from intramolecular cross-linking. In particular, higher ordered aggregates caused by inter-particle cross-linking were not detected by DLS and SEC at $\mathrm{pH}$ 7.4. Even at $\mathrm{pH} 10$, given the capacity for further coordination and cross-link, the detected size increase was unexpectedly defined. In contrast, at $\mathrm{pH} 4$, iron(III) and catechol formed predominantly a 1:1 complex, which resulted in a linear chain without any crosslinking.

While the conversion of polypeptide architectures to form intramolecular interactions was adjusted by tuning the amount of iron(III) and $\mathrm{pH}$ (Figure 2), intermolecular cross-links become dominant by increasing the concentration of the components. The formation of the hydrogels was observed by mixing 4 wt $\%$ and 8 wt $\%$ of dcHSA-PEG-C3 with water at pH 4 , 7.4 and 10 and by adding different amounts of iron(III)-solution. Twelve different protein hydrogels consisting of precisely adjusted amounts of iron(III) at different $\mathrm{pH}$ values and weight percentages were synthesized and their rheological properties were characterized

\section{(Table 1, Figure 4).}

Table 1. List of produced hydrogels (H1-12) with different amounts of iron(III), weight percentage $(\mathrm{wt} \%)$ of dcHSA-PEG-C3, pH values, including the analyzed rheological parameters storage moduli $G^{\prime}$ and loss moduli G' (strain $0.1 \%$, frequency $1 \mathrm{~Hz}$ ), the selfhealing properties (quantified by combined measurements of an oscillatory strain sweep $(0.01-$ $1000 \%$, frequency $1 \mathrm{~Hz}$ ) with subsequent oscillatory time sweep measurement (strain $0.1 \%$, frequency $1 \mathrm{~Hz})$ ), and gel-to-sol transition points (strain sweeps $(0.01-1000 \%)$, frequency $1 \mathrm{~Hz}) .(-)=$ n.d., not determined.

\begin{tabular}{|c|c|c|c|c|c|c|c|c|c|c|c|c|}
\hline & $\mathrm{H} 1$ & $\mathrm{H} 2$ & H3 & $\mathrm{H} 4$ & H5 & $\mathrm{H} 6$ & $\mathrm{H7}$ & $\mathrm{H} 8$ & $\mathrm{H9}$ & $\mathrm{H} 10$ & H11 & $\mathrm{H} 12$ \\
\hline Equiv. iron(III) & 45 & 90 & 180 & 180 & 45 & 90 & 180 & 180 & 45 & 90 & 180 & 180 \\
\hline Vol. [iron(III) sol.] [uL] & 0.5 & 1 & 2 & 4 & 0.5 & 1 & 2 & 4 & 0.5 & 1 & 2 & 4 \\
\hline $\mathbf{w t} \%$ & 4 & 4 & 4 & 8 & 4 & 4 & 4 & 8 & 4 & 4 & 4 & 8 \\
\hline $\mathrm{G}^{\prime}[\mathrm{Pa}]$ & liquid & 9 & 9 & 139 & 7 & 189 & 562 & 4359 & - & 15 & 118 & 2194 \\
\hline G" [Pa] & liquid & 4 & 1 & 10 & 3 & 37 & 92 & 573 & - & 3 & 12 & 132 \\
\hline self-healing [\%] & - & - & - & $94 \pm 8$ & - & $60 \pm 0$ & $62 \pm 1$ & $84 \pm 14$ & - & - & $81 \pm 19$ & - \\
\hline gel-sol-point [strain \%] & - & - & - & $254 \pm 1$ & - & $31 \pm 0$ & $35 \pm 0$ & $58 \pm 7$ & - & - & $118 \pm 22$ & $101 \pm 2$ \\
\hline
\end{tabular}


The mechanical properties, the storage moduli $\left(G^{\prime}\right)$ and loss moduli $\left(G^{\prime \prime}\right)$ of different gels were investigated in time-sweep experiments (Figure 4b, S5, S6, S7, Table 1) with fixed frequency and strain $(0.1 \%, 1 \mathrm{~Hz})$. No crossover point of $\mathrm{G}^{\prime}$ and $\mathrm{G}^{\prime \prime}$ was observed throughout the measurement, indicating instant gelation during sample preparation $(\sim 30 \mathrm{~s})$ and constant gel stability over time. With 4 wt\% of dcHSA-PEG-C3 and 45 equivalents of iron(III) at pH 7.4, the material began to show gel-like properties as the storage modulus $\left(\mathrm{G}^{\prime}\right)$ was higher than the loss modulus $\left(G^{\prime \prime}\right)$. Further time-sweep experiments of the gels at $\mathrm{pH} 4,7.4$, and 10, independent of the amounts of polymeric material, showed that higher amounts of iron(III) resulted in higher G', indicating a higher cross-link density. This observation is consistent with the results of the UV-Vis experiments, which also demonstrated higher cross-linking of the bisand tris-catecholato-iron(III) complexes. At $\mathrm{pH} 4$, the material demonstrated storage and loss moduli of very soft gels $\left(\mathrm{G}^{\prime} \approx 10 \mathrm{~Pa}\right)$ with liquid like behaviour $(\mathbf{H 1})$ because of the missing coordinative cross-linking structures. At $\mathrm{pH}$ 10, the gels were more robust according to their mechanical strength $\left(\mathrm{G}^{\prime} \approx 120 \mathrm{~Pa} ; \mathbf{H 1 1}\right)$ as the coordination density increases to accommodate up to three catechols (Figure 4b). Although increasing the number of interactions naturally leads to more stable structures, it is interesting to note that bis-complexes have been reported to exhibit higher mechanical strength than the tris-complexes. ${ }^{[23,24]}$ Indeed, according to the results of the storage and loss moduli, a higher mechanical strength of the bis-complexes dominated at $\mathrm{pH} 7.4$ compared to the tris-complexes at $\mathrm{pH} 10$. A higher amount of polymeric material also had a strong strengthening effect, as $\mathrm{G}^{\prime}$ and $\mathrm{G}^{\prime \prime}$ of the gels with $8 \mathrm{wt} \%$ material were 6 to 10 times higher than the gels with $4 \mathrm{wt} \%$. The gels with the highest storage moduli reached G' of around $4300 \mathrm{~Pa}$, which is comparable to the stiffness of liver tissue. ${ }^{[25]}$ The crosslinked gel was further characterized by frequency sweeps, but no frequency dependence for both $\mathrm{G}^{\prime}$ and $\mathrm{G}^{\prime \prime}$ ' between to $0.05-10 \mathrm{~Hz}$ and only a small continuous increase without intersection at higher frequencies was observed (Figure S9). 

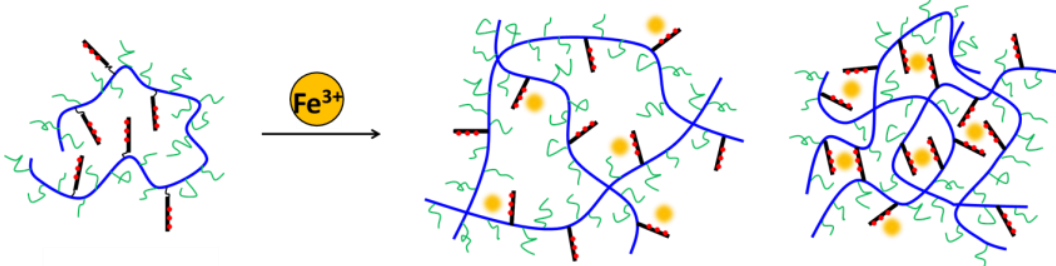

- Tunable stiffness

- Strain bearing ability

- Shear-thinning

- Thixotropic behavior

4 wt\% / 8wt\%

$\mathrm{G}^{\prime}<10^{2} \mathrm{~Pa}$

$\mathrm{G}^{\prime}<10^{4} \mathrm{~Pa}$

dcHSA-PEG-C3

$\mathrm{pH}$

b)

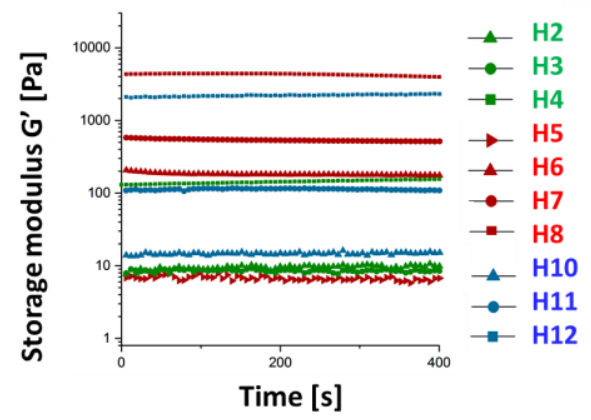

c)

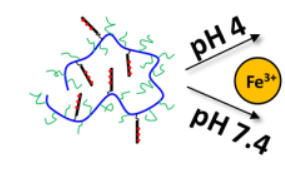

dcHSA-PEG-C3

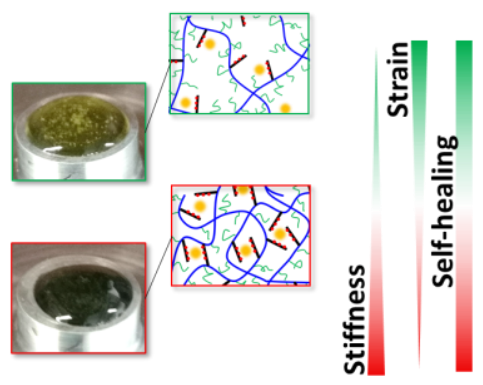

d)

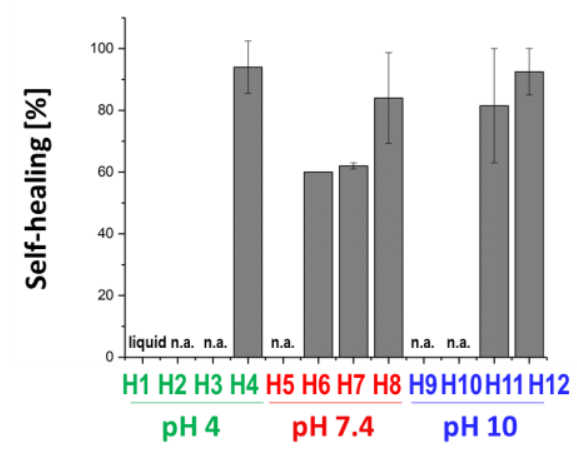

e)

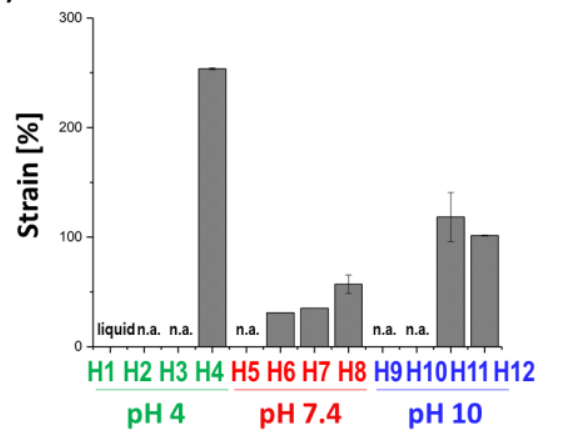

f)

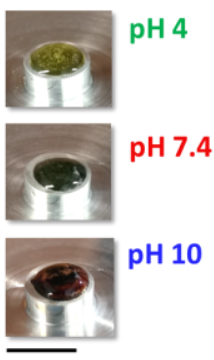

Figure 4. a) Schematic presentation of iron(III)-induced gelation of dcHSA-PEG-C3 and pH dependent cross-linking. b) Rheological measurements of produced hydrogels $(\mathrm{H})$ with different amounts of iron(III), weight percentage of dcHSA-PEG-C3 and $\mathrm{pH}$ values. Comparison of different storage moduli $\mathrm{G}^{\prime}$ with fixed strain $(0.1 \%)$ and frequency $(1 \mathrm{~Hz})$, loss moduli are listed in Figure S5-7, c) Schematic illustration of $\mathrm{pH}$ dependent iron(III)-induced gelation and the resulting adjustablility of hydrogel properties, showcasing hydrogels formed at $\mathrm{pH} 4$ (e.g., H4) or $\mathrm{pH} 7.4$ (e.g., H8) differing in their mechanical properties. d) Quantification of the self-healing properties of H1-12 (combined measurements of an oscillatory strain sweep $(0.01-1000 \%$, frequency $1 \mathrm{~Hz})$ with subsequent low strain oscillatory time sweep measurement (strain $0.1 \%$, frequency $1 \mathrm{~Hz}$ )). e) Gel-to-sol transition points of H1-12, measured by strain sweeps (0.01-1000\%) at fixed frequency of $1 \mathrm{~Hz}$. f) Fotographs of iron(III)-induced hydrogels at different $\mathrm{pH}$. Scale bar is $8 \mathrm{~mm}$. 
Subsequently, the self-healing properties of the hydrogels were evaluated by consecutive measurements of strain sweeps up to strains of $1000 \%$, followed by subsequent time sweeps with low fixed strain $(0.1 \%)$, both at a fixed frequency of $1 \mathrm{~Hz}$. Very fast recovery rates of $60 \%$ to $100 \%$ of the gel's storage moduli were observed after removal of high shear force, even though no resting period was given after each step-strain experiment (Figure 4d, Table 1, Supporting Information), indicating the possibility of minimally-invasive administration of the gels via injection. The gels at $\mathrm{pH} 4(\mathbf{H 1}-\mathbf{H 4})$ with a larger proportion of flexible and more dynamic cross-linking bonds e.g., weak electrostatic forces between catechol and iron(III), showed higher recovery rates which is comparable to known literature. ${ }^{[26]}$ It has been shown before that dynamically cross-linked polyesters showed characteristic vitrification properties and usually demonstrated quantitative self-healing features. ${ }^{[27]}$ At higher $\mathrm{pH}$ values (pH 7.4 and $\mathrm{pH}$ 10) more coordinative cross-linking occurs and high recovery rates were observed, indicating a high reversibility of the stable bis- and tris-catecholato-iron(III) complexes.

The strain values of the hydrogels (Figure 4e, Table 1), varying from 30 to 250\%, mark the gel-to-sol transition, from which the system begins to act like a liquid. The differences can be attributed to the extent of coordination of catechols towards iron(III): at $\mathrm{pH} 4$, monocomplexation dominates, whereas at $\mathrm{pH} 7.4$ and 10, higher ordered complexes favors the establishment of strong interchain coordinative bonds. In comparison to other similar catecholiron-based hydrogel systems, the gels described herein demonstrated high self-healing values without given healing time ${ }^{[21,28,29]}$ as well as the ability to bear high strain values of more than $100 \%$ up to the gel-to-sol transition (Figure 4, Table 1), which is significantly larger than iron(III)/catechol induced ${ }^{[21]}$ or telechelic bifunctional polymer-induced ${ }^{[30]}$ hydrogels from biopolymers. 


\section{Conclusion}

We have demonstrated folding of a long polypeptide chain of HSA into nanoparticles or hydrogels based on intra or intermolecular dynamic covalent bond formation and $\mathrm{pH}$ responsive iron(III)/catechol interactions. By dynamic covalent intermolecular cross-linking at low concentrations within the subnanomolar regime, single chain polypeptide nanoparticles with tunable sizes between pH 7.4 and 10 were achieved, based on the coordination level of iron(III). At high concentrations (> $4 \mathrm{wt} \%$ dcHSA-PEG-C3), where interchain coordinative crosslinking was favored, macroscopic hydrogel matrixes with varying mechanical strength from $\mathrm{G}^{\prime}, \mathrm{G}^{\prime \prime}=0 \mathrm{~Pa}$ to $\mathrm{G}^{\prime}=4.4 \mathrm{kPa}, \mathrm{G}^{\prime \prime}=0.6 \mathrm{kPa}$ were obtained by simply adjusting the $\mathrm{pH}$ and the amount of iron(III) featuring rapid gelation without toxic reagents, fast and autonomous selfhealing, tunable mechanical properties and high adaptability to local environmental conditions. Given the ease of adjustability of mechanical properties, these hydrogels could be particularly useful for biomedical approaches, where stiffness, gel-to-sol transitions, and self-healing are major key parameters for successful integration of artificial cell matrices, drug delivery platforms, and their respective application routes, such as spray, injection, or implantation, e.g., via endoscopic routes. As such, systems like $\mathbf{H 4}$ that are able to gelate in an acidic environment without toxic catalysts, could be attractive for the promotion of gastrointestinal mucosal healing, which could be of interest for the prevention of ulcer-related complications. ${ }^{[31]}$ Hydrogels like $\mathbf{H 8}$ on the other side are highly attractive as artificial cell matrices with a broad relevance in various tissue engineering approaches.

\section{Supporting Information}

Supporting Information is also available from ChemRxiv.

\section{Acknowledgements}

The authors acknowledge the financial support from the Deutsche Forschungsgemeinschaft (DFG, German Research Foundation) project number 316249678 - SFB 1279 (C01), the 
Federal Ministry of Education and Research of Germany (BMBF) in the framework of ProMatLeben Polymere (InGel-NxG; Project number 13XP5086F) and the Max Planck-Bristol Centre for Minimal Biology.

\section{Conflict of Interest}

The authors declare no conflict of interest.

[1] O. Altintas, C. Barner-Kowollik, Macromol. Rapid Commun. 2016, 37, 29.

[2] O. Altintas, C. Barner-Kowollik, Macromol. Rapid Commun. 2012, 33, 958.

[3] G. M. ter Huurne, A. R. A. Palmans, E. W. Meijer, CCS Chem. 2019, 64.

[4] D. E. Whitaker, C. S. Mahon, D. A. Fulton, Angew. Chemie Int. Ed. 2013, 52, 956.

[5] E. Verde-Sesto, A. Arbe, A. J. Moreno, D. Cangialosi, A. Alegría, J. Colmenero, J. A. Pomposo, Mater. Horizons 2020, 7, 2292.

[6] J. Li, P. Nowak, S. Otto, J. Am. Chem. Soc. 2013, 135, 9222.

[7] M. Radzinski, T. Oppenheim, N. Metanis, D. Reichmann, Biomolecules 2021, 11, 469.

[8] R. D. B. Fraser, T. P. MacRae, L. G. Sparrow, D. A. D. Parry, Int. J. Biol. Macromol. 1988, $10,106$.

[9] G. Bulaj, Biotechnol. Adv. 2005, 23, 87.

[10] I. Bosnjak, V. Bojovic, T. Segvic-Bubic, A. Bielen, Protein Eng. Des. Sel. 2014, 27, 65.

[11] P. J. Hogg, Trends Biochem. Sci. 2003, 28, 210.

[12] K. M. Cook, P. J. Hogg, Antioxid. Redox Signal. 2013, 18, 1987.

[13] W. R. Harris, C. J. Carrano, K. N. Raymond, J. Am. Chem. Soc. 1979, 101, 2213.

[14] M. Hebel, A. Riegger, M. M. Zegota, G. Kizilsavas, J. Gačanin, M. Pieszka, T. Lückerath, J. A. S. Coelho, M. Wagner, P. M. P. Gois, D. Y. W. Ng, T. Weil, J. Am. Chem. Soc. 2019, 141, 14026.

[15] A. W. Bosman, L. Brunsveld, B. J. B. Folmer, R. P. Sijbesma, E. W. Meijer, 
Macromol. Symp. 2003, 201, 143.

[16] G. Springsteen, B. Wang, Tetrahedron 2002, 58, 5291.

[17] J. Gačanin, A. Kovtun, S. Fischer, V. Schwager, J. Quambusch, S. L. Kuan, W. Liu, F. Boldt, C. Li, Z. Yang, D. Liu, Y. Wu, T. Weil, H. Barth, A. Ignatius, Adv. Healthc. Mater. 2017, 6, 1700392.

[18] J. Gačanin, J. Hedrich, S. Sieste, G. Glaßer, I. Lieberwirth, C. Schilling, S. Fischer, H. Barth, B. Knöll, C. V. Synatschke, T. Weil, Adv. Mater. 2019, 31, 1805044.

[19] D. P. Nair, M. Podgórski, S. Chatani, T. Gong, W. Xi, C. R. Fenoli, C. N. Bowman, Chem. Mater. 2014, 26, 724.

[20] A. B. Lowe, Polym. Chem. 2010, 1, 17.

[21] J. Lee, K. Chang, S. Kim, V. Gite, H. Chung, D. Sohn, Macromolecules 2016, 49, 7450.

[22] P. Sánchez, N. Gálvez, E. Colacio, E. Miñones, J. M. Domínguez-Vera, Dalt. Trans. 2005, 811 .

[23] Y. Li, J. Wen, M. Qin, Y. Cao, H. Ma, W. Wang, ACS Biomater. Sci. Eng. 2017, 3, 979.

[24] Z. Xu, Sci. Rep. 2013, 3, 2914.

[25] M. A. Green, R. Sinkus, S. C. Gandevia, R. D. Herbert, L. E. Bilston, NMR Biomed. 2012, 25, 852 .

[26] P. S. Yavvari, A. Srivastava, J. Mater. Chem. B 2015, 3, 899.

[27] W. Denissen, J. M. Winne, F. E. Du Prez, Chem. Sci. 2016, 7, 30.

[28] P. S. Yavvari, S. Pal, S. Kumar, A. Kar, A. K. Awasthi, A. Naaz, A. Srivastava, A. Bajaj, ACS Biomater. Sci. Eng. 2017, 3, 3404.

[29] C. Seidler, D. Y. W. Ng, T. Weil, Tetrahedron 2017, 73, 4979.

[30] W. Xie, Q. Gao, Z. Guo, D. Wang, F. Gao, X. Wang, Y. Wei, L. Zhao, ACS Appl. Mater. Interfaces 2017, 9, 33660. 
[31] X. Xu, X. Xia, K. Zhang, A. Rai, Z. Li, P. Zhao, K. Wei, L. Zou, B. Yang, W.-K. Wong, P. W.-Y. Chiu, L. Bian, Sci. Transl. Med. 2020, 12, eaba8014. 